Published in J. Stat. Phys, 70, 463-478 (1993).

\title{
Stochastic Resonance: Linear Response and Giant Nonlinearity
}

\author{
M.I.Dykman ${ }^{1}$, D.G.Luchinsky², R.Mannella ${ }^{3}$, \\ P.V.E.McClintock ${ }^{4}$, N.D.Stein ${ }^{4}$, and N.G.Stocks ${ }^{4}$ \\ ${ }^{1}$ Department of Chemistry, Stanford University, Stanford, CA 94305, USA. \\ 2 All-Union Research Institute for Metrological Service, 117965 Moscow, Russia. \\ 3 Dipartimento di Fisica, Universita di Pisa, \\ Piazza Torricelli 2, 56100 Pisa, Italy. ${ }^{4}$ School of Physics and Materials, Lancaster University, \\ Lancaster LA1 4YB, United Kingdom.
}

\begin{abstract}
The response of a bistable noise-driven system to a weak periodic force is investigated using linear response theory (LRT) and by analogue electronic experiment. For quasi-thermal systems the response, and in particular its increase with increasing noise intensity $D$, are described by the fluctuation-dissipation relations. For small $D$ the low-frequency susceptibility of the system $\chi(\omega)$ has been found in explicit form allowing for both forced oscillations about the stable states and periodic modulation of the probabilities of fluctuational transitions between the states. It is shown, both theoretically and experimentally, that a phase lag $\phi$ between the force and the response passes through a maximum when $D$ is tuned through the range where stochastic resonance (SR) occurs. A giant nonlinearity of the response is shown to arise for small $D$ and small frequencies of the driving force. It results in the signal induced by a sinusoidal force being nearly rectangular. The range of applicability of LRT is established.
\end{abstract}

KEY WORDS: stochastic resonance; fluctuation-dissipation relations; spectral density of fluctuations; bistable systems; fluctuational transitions; giant nonlinearity; linear response; susceptibility. 


\section{INTRODUCTION}

Stochastic resonance (SR) is a phenomenon in which, counter-intuitively, a weak periodic signal, usually in a bistable system, can be amplified by the addition of external noise. Considered initially in the context of ice-ages ${ }^{(1,2)}$, it has been observed recently in active ${ }^{(3)}$ and passive ${ }^{(4)}$ optically bistable systems, hybrid electron spin resonance (ESR) devices ${ }^{(5)}$, a magnetoelastic ribbon ${ }^{(6)}$, and also in several analogue electronic experiments ${ }^{(7-10)}$. The origin of SR in all these seemingly different systems lies in the fact that the periodic driving force modulates the probabilities of fluctuational transitions between the co-existing stable states and hence the populations of the states; in its turn this gives rise to a comparatively strong modulation of a coordinate of the system with an amplitude proportional to the distance between the stable positions ${ }^{(11)}$. Since the transitions themselves arise because of noise and the transition probabilities increase sharply (exponentially, for Gaussian noise) with the noise intensity, the efficiency of the modulation is also sharply increased. The theoretical consideration of SR has been carried out, most commonly, for a discrete two-state model or, in the case of continuous systems, was based on an approximate or numerical solution of the Fokker-Planck equation for a periodically driven system, often with contradictory results $^{(1,2,12-16)}$.

An alternative approach to SR is based ${ }^{(8)}$ on linear response theory (LRT). According to LRT, if a system with a coordinate $q$ is driven by a weak force $A \cos \Omega t$ (the addition to the Hamiltonian function of the system is of the form of $-A q \cos \Omega t$ ), there arises a small periodic term in the ensemble-averaged value of the coordinate, $\delta\langle q(t)\rangle$, oscillating at the same frequency $\Omega$ and with amplitude $a$ proportional to that of the force ${ }^{(17)}$ :

$$
\begin{gathered}
\delta\langle q(t)\rangle=a \cos (\Omega t+\phi), \quad A \rightarrow 0 \\
a=A|\chi(\Omega)|, \quad \phi=-\arctan [\operatorname{Im} \chi(\Omega) / \operatorname{Re} \chi(\Omega)]
\end{gathered}
$$

The quantity $\chi(\Omega)$ here is the susceptibility of the system. Eq.(1) holds for dissipative and fluctuating systems that do not display persistent periodic oscillations in the absence of the force $A \cos \Omega t$; it is SR in bistable systems of this kind ("conventional SR") that is considered below ("nonconventional SR" will be considered elsewhere ${ }^{(18)}$ ). The function $\chi(\Omega)$ contains, basically, all information on the response of the system to a weak driving force. It gives both the amplitude of the signal, $a$, and its phase lag with respect to the force, $\phi$. In its turn, the value of $\frac{1}{4} a^{2}$ gives the intensity (i.e., the area) of the delta-shaped spike in the spectral density of fluctuations (SDF) $Q(\omega)$ of the system at the frequency $\Omega$ of the driving force,

$$
Q(\omega)=\lim _{\tau \rightarrow \infty}(4 \pi \tau)^{-1}\left|\int_{-\tau}^{\tau} d t q(t) \exp (i \omega t)\right|^{2}
$$

The onset of such a spike follows immediately from (1) with account taken of the principle of the decay of correlations:

$$
\left\langle q(t) q\left(t^{\prime}\right)\right\rangle \rightarrow\langle q(t)\rangle\left\langle q\left(t^{\prime}\right)\right\rangle \quad \text { for } \quad\left|t-t^{\prime}\right| \rightarrow \infty
$$

Following Ref 3 the response of the system, in the context of SR, is often characterized by the ratio $R$ of the area of the above spike to the value $Q^{(0)}(\Omega)$ of the SDF at the given 


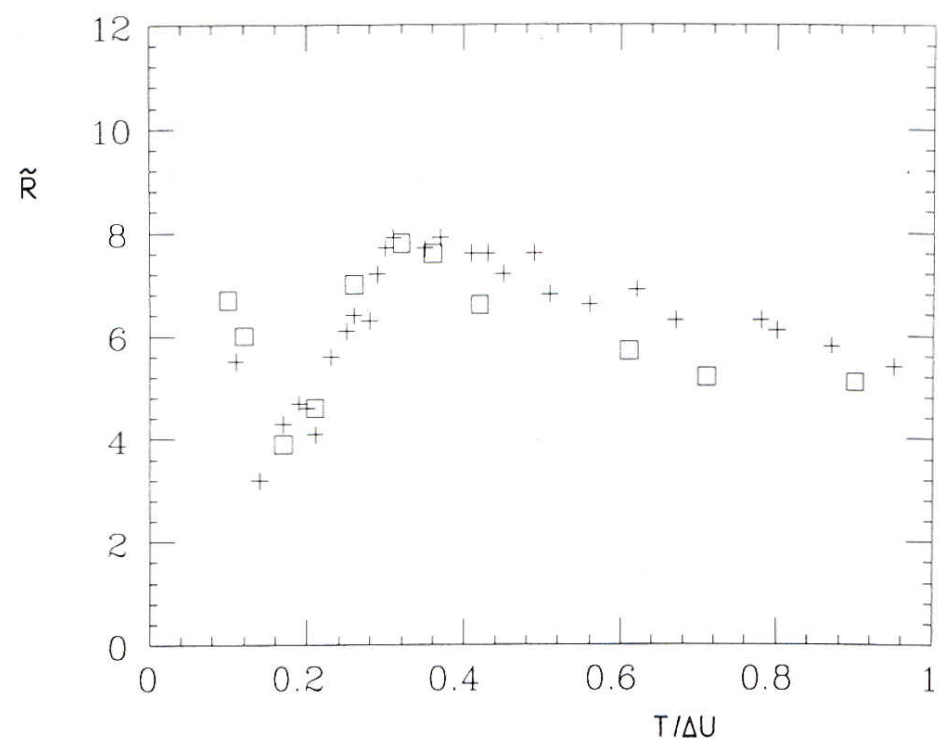

Figure 1: Signal-to-noise ratio vs. noise intensity for the Brownian motion in the double-well potential U(q) (6): $\ddot{q}+2 \Gamma \dot{q}+U^{\prime}(q)=(2 \Gamma)^{\frac{1}{2}} f(t)+A \cos \Omega t$. The values of $\tilde{R}=6.51 \times 10^{-4} R$ are given for $\Omega=0.0695, A=0.1, \Gamma=0.125$. $\square$ - Direct measurements, + - data calculated from the measured $Q^{(0)}(\omega)$ via fluctuation-dissipation relations (5).

frequency $\Omega$ in the absence of periodic driving, i.e. by the signal-to-noise ratio. It is evident from (1)-(3) that $R$ may be expressed in terms of a susceptibility $\chi(\Omega)$ :

$$
R=\frac{1}{4} A^{2}|\chi(\Omega)|^{2} / Q^{(0)}(\Omega) \quad(A \rightarrow 0)
$$

Therefore, the evolution of the susceptibility and of $Q^{(0)}(\Omega)$ with varying noise intensity $D$ show immediately whether or not SR (i.e. an increase and subsequent decrease in $R$ with increasing $D$ ) is to be expected at a given frequency.

An important advantage of describing SR in terms of the susceptibility is that such a description relates SR to standard linear-response phenomena (conductivity, magnetic susceptibility, etc) investigated in physical kinetics. One more advantage is that quite often the systems investigated are in thermal equilibrium or in quasi-equilibrium. In this case the susceptibility can be expressed immediately in terms of the $\operatorname{SDF} Q^{(0)}(\Omega)$ in the absence of periodic driving via the fluctuation-dissipation relations ${ }^{(17)}$ :

$$
\operatorname{Re} \chi(\omega)=\frac{2}{T} \mathrm{P} \int_{0}^{\infty} d \omega_{1} Q^{(0)}\left(\omega_{1}\right) \omega_{1}^{2}\left(\omega_{1}^{2}-\omega^{2}\right)^{-1}, \quad \operatorname{Im} \chi(\omega)=\frac{\pi \omega}{T} Q^{(0)}(\omega)
$$

where $\mathrm{P}$ implies the Cauchy principal part and $T$ is temperature in energy units. It follows from Eqs. (4), (5) that the onset of SR can be predicted from the purely experimental data on the evolution of the SDF of a system with temperature without assuming anything at all about the equations that describe its dynamics, i.e. for a system treated as a "black box".

The relevance of this approach to $\mathrm{SR}$ is seen from Fig.1 where some data from analog experiments $^{(8)}$ for an electronic system simulating Brownian motion in a bistable potential are shown. The system simulated is quasi-thermal equilibrium, with noise intensity $D$ 
standing for temperature $T$ in (5). The data demonstrate that $\mathrm{SR}$ in signal-to-noise ratio is described quantitatively by the fluctuation-dissipation relations (5) and Eq. (4) even in the range where the explicit analytic calculation of the susceptibility of the system was not possible.

However, it is not only signal-to-noise ratio that is important in the context of the influence of noise on the response of the system to a sinusoidal driving force. One of the purposes of the present paper is to predict, on the basis of the analysis of the susceptibility of a bistable system, and to observe experimentally a bell-shaped dependence of the phase lag $\phi$ of the signal on the noise intensity $D$. The problem of the phase shifts is considered in Sec. II below. In Sec. III we investigate the nonlinearity of the response of a bistable system to a periodic force and demonstrate, both theoretically and experimentally, the onset of an extremely strong nonlinearity for low-frequency driving. Sec.IV contains some concluding remarks.

\section{PHASE SHIFTS IN STOCHASTIC RESONANCE}

The presence or absence of phase shifts in SR is a conundrum of many years' standing. The first prediction of a phase shift seems to have been due to Nicolis ${ }^{(2)}$ who concluded that, for an overdamped system fluctuating in a bistable potential, $\phi=-\arctan \left(\Omega / W^{(0)}\right)$ where $W^{(0)}$ is the sum of the transition rates out of each of the potential wells; similar results were also obtained by McNamara and Wiesenfeld ${ }^{(12)}$. On the other hand, Gammaitoni et al., claimed ${ }^{(9)}$ that analog simulations ${ }^{(10)}$ as well as numerical computations ${ }^{(15)}$ "had ruled out [the phase shifts] as apparently spurious". Because the onset of the phase shifts follows automatically from the LRT-approach to $\mathrm{SR}^{(8)}$, Gammaitoni et al ${ }^{(9)}$ assumed this in itself to be a good reason to doubt the applicability of LRT to SR. Recently ${ }^{(5)}$, when investigating SR in a hybrid ESR system, this group did observe large phase shifts. However, the dependence of $\phi$ on the noise intensity was found to be monotonic, apparently due to the signal having been modified by passage through a two-state filter. In contrast, the dependence of $\phi$ on the noise intensity that follows from LRT for the undistorted signal, and which has been observed in the experiments reported below, is strongly nonmonotonic.

The problem of phase shifts in SR is also interesting from the viewpoint of relating SR to standard resonance phenomena ( $\mathrm{cf}^{(13)}$ ). It is well known in physics that when the frequency $\Omega$ of an external driving force is swept through the resonant frequency of a system the phase lag $\phi$ of the signal in the system decreases monotonically from nearly zero for small $\Omega$ to nearly $-180^{\circ}$ for large $\Omega$ passing through $\approx-90^{\circ}$ at the resonant value of $\Omega$. Conventional $\mathrm{SR}$ in bistable systems arises because, with increasing noise intensity, the probabilities of fluctuational transitions between the stable states become of the same order of magnitude or larger than the frequency $\Omega$ of the driving force, thereby switching on the mechanism of strong response associated with the transitions. So, the physics is different from that in a standard resonance, and the dependence of the phase lag on the noise intensity would not necessarily be expected to be the same as $\phi(\Omega)$ in a resonating system. Last but not least, the investigation of the phase shifts under SR can give an important extra argument in relation to whether ${ }^{(8)}$ or not ${ }^{(9)} \mathrm{SR}$ can properly be treated as a linear response phenomenon.

In this Section we show in considerable detail, both experimentally and theoretically, that phase shifts do indeed accompany SR; however, in continuous systems, they take a form completely different from that predicted for two-state systems ${ }^{(2)}$. We treat the simplest nontrivial system: an overdamped Brownian particle moving in a symmetric bistable 


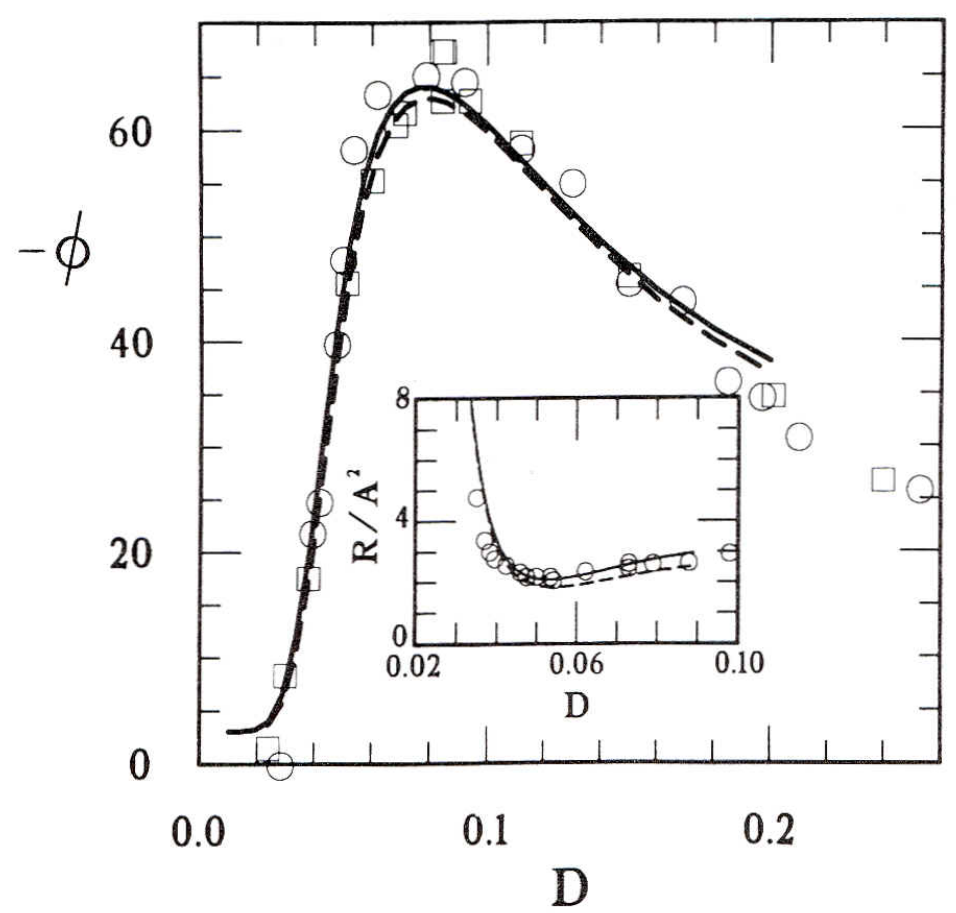

Figure 2: The phase shift $-\phi$ (degrees) between the periodic force of amplitude $A$ and the averaged coordinate $\langle q(t)\rangle$ of the overdamped bistable system (6) measured as a function of noise intensity $D$ in the electronic experiment for $\Omega=0.1$ and: $A=0.04$ (circles); $A=$ 0.2 (squares). The dashed curve represents the theoretical prediction based on LRT and the fluctuation dissipation theorem; the full curve takes account of nonlinear corrections for $A$ $=0.04$. The inset shows the normalized signal-to-noise ratio in the region of the minimum in $R$.

potential and, in addition, driven periodically,

$$
\dot{q}+U^{\prime}(q)=A \cos \Omega t+f(t), \quad U(q)=-\frac{1}{2} q^{2}+\frac{1}{4} q^{4}
$$

where $f(t)$ is zero-mean Gaussian noise of intensity $D$,

$$
\left\langle f(t) f\left(t^{\prime}\right)\right\rangle=2 D \delta\left(t-t^{\prime}\right)
$$

In the absence of periodic forcing the system (6), (7), irrespective of the particular form of the (confining) potential $U(q)$, is quasi-thermal: its distribution over energy $U(q)$ (an overdamped system has potential energy only) is Gibbsian, with temperature

$$
T=D .
$$

Therefore, the fluctuation-dissipation relations (5) hold and, for weak periodic driving force, i.e., for small $A$ in (6), the susceptibility $\chi(\Omega)$ can be expressed in terms of the spectral density of fluctuations $Q^{(0)}(\omega)$ for $A=0$. Explicit expressions for $Q^{(0)}(\omega), \chi(\omega)$ can be obtained analytically for small noise intensities (low temperatures) $D \ll \Delta U$ where, for a general double-well potential, $\Delta U$ is the depth of the (shallower) potential well, and $\Delta U=\frac{1}{4}$ for the 
model (6). In this range $Q^{(0)}(\omega)$ and $\chi(\omega)$ are given ${ }^{11,19}$ by the sums of partial contributions from fluctuations about the equilibrium positions $q_{n},\left[U^{\prime}\left(q_{n}\right)=0, U^{\prime \prime}\left(q_{n}\right)>0, n=\right.$ 1,$2 ; q_{n}=(-1)^{n}$ for the potential (6)] and of the contribution from interwell transitions,

$$
Q^{(0)}(\omega)=\sum_{n=1,2} w_{n} Q_{n}^{(0)}(\omega)+Q_{t r}^{(0)}(\omega), \quad \chi(\omega)=\sum_{n=1,2} w_{n} \chi_{n}(\omega)+\chi_{t r}(\omega)
$$

Here, $w_{n}$ is the population of the $n$-th stable state. The susceptibilities $\chi_{n}(\omega)$ and $\chi_{t r}(\omega)$ are expressed in terms of $Q_{n}^{(0)}(\omega), Q_{t r}^{(0)}(\omega)$ by Eq.(5), and therefore only the spectral densities of fluctuations will be written down below in explicit form. For the model $(6), w_{1}=w_{2}=$ $\frac{1}{2}, Q_{1}^{(0)}(\omega)=Q_{2}^{(0)}(\omega)$ and $\chi_{1}(\omega)=\chi_{2}(\omega)$.

The SDF for the intrawell vibrations $Q_{n}^{(0)}(\omega)$ can be obtained by expanding $U(q)$ about the equilibrium position $q_{n}$. Assuming the nonlinear terms small, and allowing for them by perturbation theory ${ }^{19}$, one obtains

$$
\begin{gathered}
Q_{n}^{(0)}(\omega) \simeq L_{n}(\omega)-\pi L_{n}^{2}(\omega)\left[U_{n}^{(I V)}-9 U_{n}^{\prime \prime \prime 2} U_{n}^{\prime \prime}\left(4 U_{n}^{\prime \prime 2}+\omega^{2}\right)^{-1}\right] \\
L_{n}(\omega)=\frac{1}{\pi} D\left(U_{n}^{\prime \prime 2}+\omega^{2}\right)^{-1}
\end{gathered}
$$

where all derivatives $U_{n}^{(k)} \equiv U^{(k)}\left(q_{n}\right)$ are evaluated for $q=q_{n}$. The contribution from interwell transitions is

$$
\begin{gathered}
Q_{t r}^{(0)}(\omega)=\frac{1}{\pi} w_{1} w_{2}\left(\langle q\rangle_{1}^{(0)}-\langle q\rangle_{2}^{(0)}\right)^{2} W^{(0)} /\left(W^{(0)^{2}}+\omega^{2}\right) \quad\left(\omega \ll U_{1,2}^{\prime \prime}\right) \\
W^{(0)} \equiv W^{(0)}(D)=W_{12}^{(0)}+W_{21}^{(0)}, \quad\langle q\rangle_{n}^{(0)}=q_{n}-\frac{1}{2} D U_{n}^{\prime \prime \prime}\left(U_{n}^{\prime \prime}\right)^{-2}
\end{gathered}
$$

Here, $\langle q\rangle_{n}^{(0)}$ is the average value of the coordinate in the n-th well neglecting interwell transitions and $W_{n m}^{(0)}$ is the probability of the transition $n \rightarrow m$ in the absence of periodic forcing (corrections $\sim D / \Delta U$ to the Kramers expression for the transition probabilities ${ }^{(20)}$ are required in (10)). In deriving (10) we have utilized the inequality $W^{(0)} \ll \Omega_{r}=\min \left(U_{1,2}^{\prime \prime}\right)$, implying that the transition probabilities are very much smaller than the relaxation rate of the system $\Omega_{r}$ (a condition that is necessary for the concept of a transition between well-defined metastable states to be meaningful).

To lowest order in $D / \Delta U$, to zeroth order in $\Omega / \Omega_{r}$, but for arbitrary $\Omega / W^{(0)}$, the expressions for the signal-to-noise ratio $R(4)$ and for the phase lag $\phi(2)$ resulting from (5), (8)-(10) for the model (6) become:

$$
\begin{gathered}
R=\frac{\pi A^{2}}{4 D^{2}}\left(\Omega_{r}^{2} W^{(0) 2}+\Omega^{2} D^{2}\right) /\left(\Omega_{r}^{2} W^{(0)}+\Omega^{2} D\right), \quad \Omega, D \ll \Omega_{r}, \quad W^{(0)} \ll D \\
\phi=-\arctan \left[\left(\Omega / \Omega_{r}\right)\left(\Omega_{r}^{2} W^{(0)}+\Omega^{2} D\right) /\left(\Omega_{r} W^{(0) 2}+\Omega^{2} D\right)\right]
\end{gathered}
$$

where $\Omega_{r}=U_{1}^{\prime \prime}=U_{2}^{\prime \prime}=2$. For very small $D$, where $W^{(0)} \ll\left(\Omega^{2} / \Omega_{r}^{2}\right) D$, it follows from $(11)$ that $R \simeq \pi A^{2} / 4 D, \quad \phi \simeq-\Omega / \Omega_{r}$. Thus, for a fixed forcing frequency $\Omega, R$ decreases with increasing $D$, whereas $\phi$ remains small and nearly independent of $D$.

For larger values of $D$, on the other hand, 


$$
\begin{gathered}
R=\left(\pi A^{2} / 4 D^{2}\right)\left[W^{(0)}+\left(\Omega^{2} D^{2} / \Omega_{r}^{2} W^{(0)}\right)\right] \\
\phi=-\arctan \left[\Omega \Omega_{r} W^{(0)} /\left(\Omega_{r} W^{(0) 2}+\Omega^{2} D\right)\right] \\
D \gg W^{(0)} \gg\left(\Omega^{2} / \Omega_{r}^{2}\right) D
\end{gathered}
$$

The behaviour of $R$ and $\phi$ as given by (13) depends on the ratio $\alpha=W^{(0)} \Omega_{r} / \Omega D$. When it is small, $R$ is sharply decreasing while $|\phi|$ is sharply increasing with increasing $D$,

$$
\phi \simeq-\alpha \equiv-W^{(0)} \Omega_{r} / \Omega D, \quad \alpha \ll 1
$$

For $\alpha \simeq 1, R$ passes its minimum and then increases with $D$ (i.e., with $\alpha$ ) up to comparatively large $D \sim \Delta U$ where the weak-noise approximation (8)-(10) is inapplicable. It is this increase that is associated with conventional SR.

The central interest of the present section relates, however, to the behaviour of $\phi$. It follows from (13) that the sharp increase of $|\phi|$ with increasing $D(14)$ saturates in the range $\alpha \gg 1$, and, for $D \equiv D_{\max } \ll \Delta U,|\phi|$ reaches its maximum:

$$
(-\phi)_{\max }=\arctan \left(\frac{1}{2}\left(\Omega_{r} / D_{\max }\right)^{\frac{1}{2}}\right), \quad W^{(0)}\left(D_{\max }\right)=\Omega\left(D_{\max } / \Omega_{r}\right)^{\frac{1}{2}}
$$

(note that, in contrast to the behaviour of $R$ vs $D$, the weak-noise theory (8)-(10) holds in the vicinity of the maximum of $|\phi|$; we stress that it is the approximation (8)-(10) that fails for strong noise, not LRT). The decrease of $-\phi$ for $D>D_{\max }$ is seen from (13) to be much less steep than the increase described by Eq. (14). Overall, it follows from (12), (13) (see also Fig. 2 where $\phi$ vs $D$ as given by (12) is plotted) that the phase shift displays a resonance-type (nonmonotonic) behaviour as a function of the noise intensity $D$. This prediction is in contrast with the earlier theories ${ }^{(2,12)}$ for two-state systems displaying SR in the signal-to-noise ratio, but exhibiting a monotonic dependence of $|\phi|$ on $D$; the phase shift in these theories is described by Eq. (13) with $\Omega_{r}$ set equal to $\infty$ (if the intrawell relaxation was infinitely fast the intrawell motion would not come into play and the system would behave as a two-state one):

$$
(\phi)_{\text {two-state }}=-\arctan \left[\Omega / W^{(0)}\right]
$$

The LRT predictions (12), (13) have been tested by means of an electronic experiment, using a circuit of conventional design ${ }^{(21)}$ to model (6). It is immediately evident from the measurements (Fig. 2), first, that contrary to ${ }^{(9,10,15)}$ large phase shifts do indeed occur as $D$ is varied and, secondly, that the LRT prediction describes the data remarkably well. For (6), with the parameters used in the experiment, a maximum value of $-\phi$ is predicted by LRT to be equal to $68^{\circ}$ and to occur at $D_{\max }=0.08$, which is to be compared with the experimental observation for $A=0.04$ of $(-\phi)_{\max }=(66 \pm 2)^{\circ}$ at $D=0.08 \pm 0.01$. In accordance with the LRT prediction, the decrease of $|\phi|$ for $D>D_{\max }$ is much more gradual than the rapid increase seen below $D_{\max }$. The measured $\phi$ is relatively insensitive to $A$ for the chosen frequency $\Omega / \Omega_{r}$ (nonlinear effects under SR are discussed in Sec. III below).

The physical origin of the nonmonotonic behaviour of the phase lag with the increasing noise intensity $D$ can be readily understood if one notices that, for very small $D \ll \Delta U$, the 
system is effectively confined to a single well and $\phi$ would be expected to be small because $\Omega$ is small compared with the reciprocal characteristic time of intrawell motion; in the opposite limit of very large $D \gg \Delta U$ the double-well character of the potential becomes irrelevant and $\phi \simeq 0$, for the same reason; so, at the intermediate values of $D$ where the interwell transitions play a substantial role and their probabilities are of the order of the frequency $\Omega$, so that the field modulates the populations of the states effectively, a phase lag associated with this modulation must inevitably give rise to a maximum in $|\phi|$, just as observed. We note that this behaviour and the decrease in the signal-to-noise ratio for very small $D$ are both related to the continuity of the system, and they are not described by a two-state theory.

The observation of the phase lags for SR in bistable systems shows explicitly the difference between this "conventional" SR and standard resonance: because $\Omega$ does not match any internal characteristic vibrational frequency of the system ${ }^{(13)}$, it should only be of the same order of magnitude or less than the Kramers hopping frequency. This is to be contrasted with SR in underdamped monostable systems ${ }^{(18,22)}$, which is a true resonance phenomenon where external noise is used to tune the natural oscillation frequency of the system to that of the periodic force. The differences between stochastic resonance and a standard (e.g. mechanical) resonance are summarised in Fig 3.

The excellent agreement between the LRT prediction and the experimental phase lag measurements in Fig.2 can be taken as an extra vindication of our suggestion ${ }^{(8)}$ that LRT provides a useful approach to the SR problem. In addition to the appealing simplicity and elegance of the linear response formalism and to the fact that LRT makes it possible to describe explicitly, allowing for intrawell vibrations, the phase shifts and the signal-tonoise ratio $R$ at small $D$, this approach has a number of other advantageous features. In particular, (a) for systems that are in thermal equilibrium or quasi-equilibrium, LRT makes it possible to predict the onset of SR solely on the basis of experimental measurements of the spectral density of fluctuations in the absence of periodic driving $Q^{(0)}(\omega)$ and its evolution with temperature (noise intensity), even in cases for which the response cannot be calculated (e.g. because there is no simple theoretical model of the system under study); (b) LRT is as easily applied to underdamped systems ${ }^{(8)}$ as it is to overdamped systems such as (6); (c) the predictive power of the LRT approach is rather strong: this approach has led, for example, to the observation ${ }^{(18)}$ of quite new kinds of SR in diverse classes of systems that differ markedly from the conventional SR system with two static stable states which is driven additively by noise and periodic force.

It seems puzzling that, despite these manifest advantages, the applicability of LRT, which is generally recognized in other areas of physics, is seriously doubted ${ }^{(9,15)}$ when SR is considered. The only limitation on the applicability of LRT is posed by the amplitude of the driving force: it should not be too large. The features of the nonlinear response of bistable systems and the range of applicability of LRT are considered in the next Section.

\section{GIANT NONLINEARITY OF LOW-FREQUENCY RESPONSE OF BISTABLE SYSTEMS}

An intriguing feature of fluctuating bistable systems is that they provide the possibility of observing a strongly nonlinear response to a comparatively weak low-frequency driving force. This possibility arises ${ }^{(8)}$ because the force modulates the probabilities $W_{n m}$ of fluctuational transitions between the states. In a quite general case where the noise driving a system 


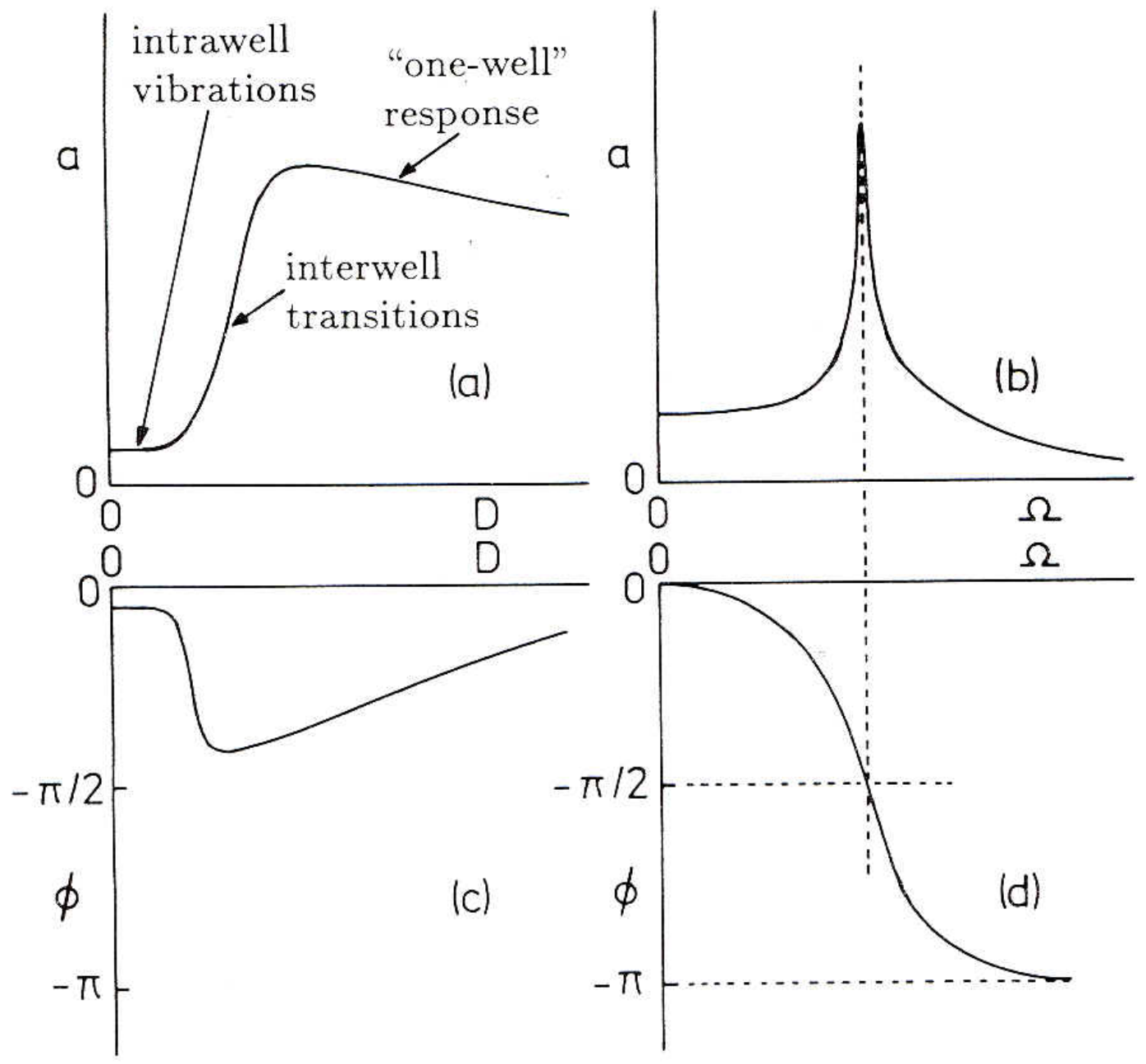

Figure 3: Comparison (schematic) of stochastic resonance with a standard (e.g. mechanical) resonance. (a) In SR, the response $a$ of the system exhibits a maximum when plotted as a function of noise intensity $D$; (b) in a standard resonance, the response $a$ shows a maximum when plotted as a function of the driving frequency $\Omega$; (c) the phase lag $-\phi$ in SR varies nonmonotonically with $D$, and the maximum lag does not necessarily coincide with the maximum in $a(D)$; (d) the phase lag - $\phi$ in a standard resonance varies monotonically with $\Omega$ and is equal to $+\pi / 2$ where $s(\Omega)$ passes through its resonance maximum. The different processes responsible for creating the SR maximum are indicated in (a).

is Gaussian (but not necessarily white), the transition probabilities $W_{n m}^{(0)}$ in the absence of periodic forcing are proportional ${ }^{(23)}$ to $\exp \left(-\mathcal{R}_{n} / \mathcal{D}\right)$, where $\mathcal{D}$ is the characteristic noise intensity, and the strength of the modulation of $W_{n m}$ is determined by the relation between the force-induced change of the "activation energy" of the transition $\mathcal{R}_{n}$ and the noise intensity $\mathcal{D}$. Obviously, this relation can be large for small $\mathcal{D}$ even if the force itself is small.

In the case of low-frequency and comparatively weak force $A \cos \Omega t$ the modulation of the activation energies is "parametrical": the transition probabilities $W_{n m}$ are determined by the instantaneous value of the force: 


$$
W_{n m} \equiv W_{n m}(t)=W_{n m}^{(0)} \exp \left(g_{n} \cos \Omega t\right), \quad g_{n}=\tilde{g}_{n} A / \mathcal{D}, \quad \Omega \ll \Omega_{r}
$$

Here, $\Omega_{r}$ is the relaxation rate of the system, and the quantities $\tilde{g}_{n}$ are equal to the derivatives $-\left(\partial \mathcal{R}_{n} / \partial A\right)_{A=0}\left(\mathcal{R}_{n}(A)\right.$ is the value of the activation energy of the transition from the state $n(n=1,2)$ of a system driven by a static force $A)$. Since the force is assumed weak, only the term linear in $A$ is taken into account in the exponent in (16). At the same time, the parameters $g_{1,2}$ are not assumed small, because they are determined by the ratio of $A$ to the noise intensity $\mathcal{D}$, and $\mathcal{D}$ is itself small. In the particular case of overdamped motion in a symmetrical double-well potential described by Eq. (6)

$$
g_{1}=-g_{2}=A / D
$$

The periodic modulation of the transition probabilities results in a modulation of the instantaneous values of the populations $w_{1,2}(t)$ of the stable states, i.e. in the periodic redistribution of the system over the states (i.e., over the potential wells or, more generally, the regions of the phase space adjacent to the attractors). This redistribution is described by the balance equation:

$$
\dot{w}_{1}(t)=-\left(W_{12}+W_{21}\right) w_{1}(t)+W_{21}, \quad w_{2}(t)=1-w_{1}(t)
$$

In the limit $\left|g_{1,2}\right| \ll 1$ Eqs.(16),(18) go over into the LRT result. In the opposite limit, $\left|g_{1,2}\right| \gg 1$, the integral that gives the steady solution of $(18) w_{1}^{(s)}(t)$,

$$
w_{1}^{(s)}(t)=\int_{-\infty}^{t} d \tau W_{21}(\tau) \exp \left(-\int_{\tau}^{t} d \tilde{\tau}\left[W_{12}(\tilde{\tau})+W_{21}(\tilde{\tau})\right]\right)
$$

can be evaluated $^{(8)}$ by the steepest descent method. The time dependence of the populations in this case is of the form of a rectangular wave. In particular, for the model (6),

$$
\begin{gathered}
w_{1}^{(s)}(t) \equiv 1-w_{2}^{(s)}(t)=-\bar{w} \sum_{n=-\infty}^{\infty}\left[\theta\left(t-\frac{2 \pi n}{\Omega}\right)-\theta\left(t-\frac{\pi(2 n+1)}{\Omega}\right)\right]+\frac{1}{2}(1-\bar{w}), \\
\bar{w}=\tanh \lambda, \quad \lambda=(2 \pi D / A)^{\frac{1}{2}}\left(W_{12}^{(0)} / 2 \Omega\right) \exp (A / D), \quad A \gg D
\end{gathered}
$$

The parameter $\bar{w}$ in (20) determines the amplitude of the rectangular wave. Note that for even large $A / D$ this parameter can be small if the frequency of the driving force exceeds strongly the transition probabilities $W_{n m}^{(0)}$, and the latter are exponentially small for small $D$. On the other hand, for $\lambda>1.5$ the value of $\bar{w} \simeq 1$, i.e., the populations of the stable state change periodically from nearly 1 to nearly 0 .

The rectangular modulation can be easily understood qualitatively by noting that, for large $\left|g_{1,2}\right|$, a transition from a given state $n$ happens, with an overwhelming probability, within a time $\sim \Omega^{-1}\left|g_{n}\right|^{-\frac{1}{2}}$ when the potential well is at its shallowest. This time is short compared with the period $2 \pi / \Omega$ of the field. Of course, the modulation comes into play if the probability of the transition over this time, i.e., the ratio $W_{n m}^{(0)} \exp \left(\left|g_{n}\right|\right) / \Omega g_{n}^{\frac{1}{2}}$ is not small.

In the general case of arbitrary $g_{n}$ Eq.(18) can be analysed numerically. To find the steady solution $w_{1,2}^{(s)}(t)$ it is convenient, allowing for its periodicity and using a standard ${ }^{(24)}$ 
expansion of the cosine exponent in (16), to change from the differential equation (18) to the set of difference equations for the Fourier-components $w_{n k}$ of $w_{n}^{(s)}(t)\left(w_{2 k} \equiv \delta_{k, 0}-w_{1 k}\right)$ :

$$
\begin{gathered}
w_{1}^{(s)}(t)=\sum_{k=-\infty}^{\infty} w_{1 k} \exp (i k \Omega t) \\
{\left[i k \Omega+W_{12}^{(0)} I_{0}\left(g_{1}\right)+W_{21}^{(0)} I_{0}\left(g_{2}\right)\right] w_{1 k}+\sum_{s \neq 0}\left[W_{12}^{(0)} I_{s}\left(g_{1}\right)+W_{21}^{(0)} I_{s}\left(g_{2}\right)\right] w_{1 k-s}=W_{21}^{(0)} I_{k}\left(g_{2}\right)}
\end{gathered}
$$

Here, $I_{n}$ are Bessel functions of imaginary argument ${ }^{(24)}$.

Because of the non-sinusoidal time dependence of the populations of the states, the time dependence of the average value of the coordinate of the system also becomes non-sinusoidal and is not described by the simple expression (1). In the general case it is of the form

$$
\delta\langle q(t)\rangle=\sum_{n \geq 0} a(n) \cos (n \Omega t+\phi(n))
$$

(we do not consider the case where vibrations occur at subharmonics: from the viewpoint of dynamics the periodic force is weak, and the nonlinearity arises only via the interplay of periodic and random forcing). It is seen from Eqs. (3), (22) that the spectral density of fluctuations of the coordinate in the nonlinear regime contains $\delta$-shaped spikes not only at the frequency $\Omega$, but also at the overtones $n \Omega, n \geq 2$. The intensity (area) of the spike at frequency $n \Omega$ is equal to $\frac{1}{4} a^{2}(n)$.

For small noise intensities and a dynamically weak periodic force, the forced vibrations of the system can still (cf. Eq. (8)) be described as a superposition of small-amplitude sinusoidal vibrations about the stable states and non-sinusoidal, generally speaking, vibrations due to the modulation of the populations of the states. Therefore,

$$
\delta\langle q(t)\rangle=A \sum_{n=1,2} w_{n 0} \operatorname{Re}\left[\chi_{n}(\Omega) \exp (-i \Omega t)\right]+\left(\langle q\rangle_{1}^{(0)}-\langle q\rangle_{2}^{(0)}\right) \operatorname{Re} \sum_{k \neq 0} w_{1 k} \exp (i k \Omega t)
$$

The partial susceptibilities $\chi_{n}(\omega)$ for overdamped motion (6) are given by the linear-response weak-noise-limit relations (5), (8), whereas the Fourier-components $w_{1 k}$ of the population of the state 1 are given by Eq. (21). These equations also give immediately the values of the amplitudes $a(n)$ and phases $\phi(n)$ of the harmonics in (22). We note that the expressions for the amplitude $a \equiv a(1)$ and phase $\phi \equiv \phi(1)$ of the vibrations at the eigenfrequency can be written in the form $(2)$ with the susceptibility $\chi(\Omega)$ replaced by

$$
\tilde{\chi}(\Omega)=w_{10} \chi_{1}(\Omega)+\left(1-w_{10}\right) \chi_{2}(\Omega)+2 A^{-1} w_{11}^{*}\left(\langle q\rangle_{1}^{(0)}-\langle q\rangle_{2}^{(0)}\right)
$$

The quantity $\tilde{\chi}(\Omega)$ should be substituted for $\chi(\Omega)$ into the expression (4) for the signalto-noise ratio at the eigenfrequency of the periodic field to describe the effects on $R$ of the nonlinearity related to modulation of the populations of the stable states.

A sample of numerical results for $R$ and $\phi$ that demonstrates the effects of nonlinearity is shown in Fig.2. The comparison with the predictions of the linear-response theory shows that, for the value of the frequency $\Omega$ chosen, these effects are small. It is important to note 


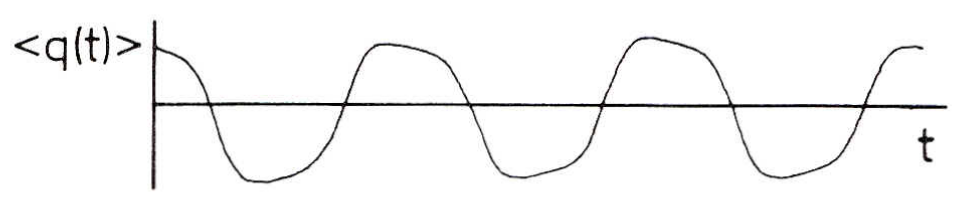

Figure 4: The averaged coordinate $\langle q(t)\rangle$ measured for the electronic circuit simulating the overdamped system (6) with $\Omega=1.9 \times 10^{-5}, A=0.1, D=0.0161$. As predicted, the results approximate a square wave. The deviations are due to intrawell vibrations, primarily.

that the degree to which the response is nonlinear depends, not only on the amplitude of the periodic force (obviously, nonlinearity is always large for a dynamically-strong force when the amplitude of the forced vibrations about the stable states is of the order of the distance between the states), but also on the frequency. This is seen from Eqs.(20), (21), but can also be readily understood from the physical picture of the effect. Of course, the ratio of the amplitude of the force-induced modulation of the activation energies of the transitions to the noise intensity is large for very small noise intensities. But the point is that the corresponding nonlinearity comes into play provided that the transitions occur with significant probability within one period of the force; otherwise the effect gets averaged out in symmetrical situation of the type (6). We note that, in the case of an asymmetric potential or attractors of more complicated origin, the ratio of the averaged-over-one-period probabilities of the transitions $\bar{W}_{12} / \bar{W}_{21}$ differs strongly from $W_{12}^{(0)} / W_{21}^{(0)}$ for $\left|g_{1,2}\right| \gg 1$ in (16). Therefore even in the range where the periodic modulation of the populations is small the change of their average values influences the response substantially, since the weighting factors for the contributions from the forced vibrations about the two stable states (the factors $w_{10}, 1-w_{10}$ in (24)) are changed as compared with their zero-field values. However, the amplitudes of these vibrations are themselves small.

To seek the onset of the giant nonlinearity of the response to a low-frequency driving force analog electronic experiments were performed with the circuit simulating (6), but now for much lower frequencies $\Omega$ than these used when just the phase shifts were being investigated (see Sec. II above). It would be expected for the system (6) that, when (20) holds, the signal resulting from sinusoidal driving should be nearly rectangular,

$$
\langle q(t)\rangle \simeq 1-2 w_{1}^{(s)}(t)
$$

where $w_{1}^{(s)}(t)$ is given by (20). A signal approximating just such a shape has indeed been observed (see Fig. 4), and for comparatively small amplitudes of the force (the data in Fig.3 refer to $A=0.1$ ). The distortion of the observed shape of the signal with respect to a rectangular one is due to the contribution from the vibrations about attractors.

\section{CONCLUSIONS}

It follows from the above results that phase lags indeed occur in SR and, for conventional SR in bistable systems, they display a bell-shaped dependence on the noise intensity. The position of the maximum of the phase shift occurs for smaller values of the noise intensity $D$ than the position of the maximum of the signal-to-noise ratio $R$. The excellent agreement obtained between experiment and the LRT prediction of the phase lag strongly supports the contention ${ }^{(8)}$ that SR may properly, and usefully, be considered as a linear response 
phenomenon. For thermal equilibrium and quasi-thermal systems the LRT-analysis can be done on the basis of the fluctuation-dissipation theorem.

A particularly interesting feature of SR in bistable systems, outside the domain of LRT, is that their response can display a giant nonlinearity. In particular, the shape of the signal induced by a low-frequency sinusoidal force can differ drastically from a sinusoid even for small amplitudes of the force. Apart from its purely scientific interest the strong nonlinearity is of potential importance in relation to various applications; we would mention, in particular, noise-protected heterodyning.

We acknowledge valuable discussions with A. R. Bulsara and J. Ross. One of us (MID) is grateful to J.Ross for extremely warm hospitality in Stanford University. The work was supported by the Ukrainian Academy of Sciences, by the United Kingdom Science and Engineering Research Council, and by the European Commission under SC1-CT91-0697(TSTS).

\section{REFERENCES}

1. R.Benzi, A.Sutera, and A.Vulpiani, J. Phys. A 14, L.453 (1981); R.Benzi, G.Parisi, A.Sutera, and A.Vulpiani, Tellus 34, 10 (1982).

2. C.Nicolis, Tellus 34, 1 (1982).

3. B.McNamara, K.Wiesenfeld, and R.Roy, Phys. Rev. Lett. 60, 2626 (1988).

4. M.I.Dykman, A.L.Velikovich, G.P.Golubev, D.G.Luchinsky, and S.V.Tsuprikov, JETP Letters 53, 193 (1991).

5. L.Gammaitoni, M.Martinelli, L.Pardi, and S.Santucci, Phys. Rev. Lett. 67, 1799 (1991); L.Gammaitoni, F.Marchesoni, M.Martinelli, L.Pardi, and S.Santucci, Phys. Lett. A 158, 449 (1991).

6. W.L.Ditto et al., J. Stat. Phys., this issue.

7. S.Fauve and F.Heslot, Phys. Lett. A 97, 5 (1983); G.Debnath, T.Zhou, and F.Moss, Phys. Rev. A 39, 4323 (1989); L.Gammaitoni, F.Marchesoni, E.Menichella-Saetta, and S.Santucci, Phys. Rev. Lett. 62, 349 (1989); G.Vemuri and R.Roy, Phys Rev. A 39, 4668 (1989).

8. M.I.Dykman, R.Mannella, P.V.E.McClintock, and N.G.Stocks, Phys. Rev. Lett. 65, 2606 (1990); M.I.Dykman, P.V.E. McClintock, R.Mannella, and N.G.Stocks, Soviet Phys. JETP Lett. 52, 144 (1990).

9. L.Gammaitoni, F.Marchesoni, E.Menichella-Saetta, and S.Santucci, Phys. Rev. Lett. 65, 2607 (1990).

10. C. Presilla, F. Marchesoni, and L. Gammaitoni, Phys. Rev. A 40, 2105 (1989); L. Gammaitoni, E. Menichella-Saetta, S. Santucci, F. Marchesoni, and C. Presilla, Phys. Rev. A 40, 2114 (1989).

11. M.I.Dykman and M.A.Krivoglaz, Soviet Phys. JETP 50, 30 (1979); M.I.Dykman and M.A.Krivoglaz, in Soviet Physics Reviews, ed I.M.Khalatnikov (Harwood, New York, 1984) vol.5, p.265. 
12. B.McNamara and K.Wiesenfeld, Phys. Rev. A 39, 4854 (1989).

13. R.F.Fox, Phys. Rev. A 39, 4148 (1989).

14. Hu Gang, G.Nicolis, and C.Nicolis, Phys.Rev. A 42, 2030 (1990).

15. P.Jung and P.Hanggi, Phys. Rev. A 41, 2977 (1990).

16. P.Jung and P.Hanggi, Phys. Rev. A 44, 8032 (1991).

17. L.D.Landau and E.M.Lifshitz, Statistical Physics, 3rd edn, part 1 (Pergamon, New York, 1980).

18. M. I. Dykman, D. G. Luchinsky, R. Mannella, P. V. E. McClintock, N. D. Stein, and N. G. Stocks, "Non-conventional stochastic resonance" J.Stat.Phys., the same issue.

19. M.I.Dykman, M.A.Krivoglaz, and S.M.Soskin, in Noise in Nonlinear Dynamical Systems, ed. F.Moss and P.V.E. McClintock (CUP, Cambridge, 1989) vol. 2, p. 347.

20. H.Risken, The Fokker-Planck Equation, 2nd edn. (Springer, Berlin, 1989).

21. L.Fronzoni, in Noise in Nonlinear Dynamical Systems (Ref 19) vol 3, p 222; and P.V.E. McClintock and F.Moss in loc. cit., vol 3, p 243.

22. N.G.Stocks, N.D.Stein, and P.V.E. McClintock, "Stochastic resonance in monostable systems", submitted to Phys. Rev. Lett.

23. M.I.Dykman, Phys. Rev. A 42, 2020 (1990).

24. Handbook of Mathematical Functions, eds. M.Abramovitz and I.M. Stegun (Dover, New York, 1970). 


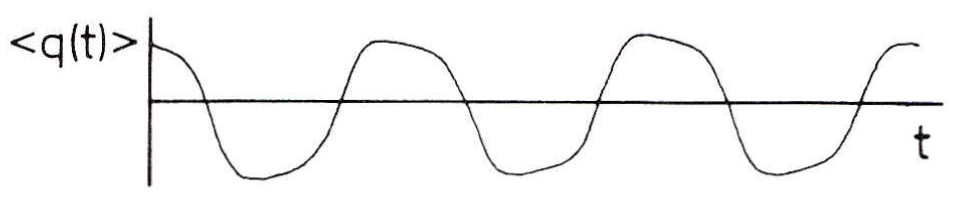

Figure 5: The averaged coordinate $\langle q(t)\rangle$ measured for the electronic circuit simulating the overdamped system (6) with $\Omega=1.9 \times 10^{-5}, A=0.1, D=0.0161$. As predicted, the results approximate a square wave. The deviations are due to intrawell vibrations, primarily. 